\title{
Shale Dynamic Properties \& Anisotropy under Triaxial Loading
}

\author{
Joël Sarout, Laurent Molez Yves Guéguen \\ Laboratoire de Géologie, Ecole Normale Supérieure, Paris, France.
}

Nasser Hoteit

Agence Nationale pour la gestion des Déchets RAdioactifs, France.

This paper is concerned with the experimental identification of the whole dynamic elastic stiffness tensor of a transversely isotropic clayrock from a single cylindrical sample under loading. Measurement of elastic wave velocities (pulse at $1 \mathrm{MHz}$ ), obtained under macroscopically undrained triaxial loading conditions are provided. Further interpretation of the velocity measurements is performed in terms of (i) dynamic elastic parameters ; and (ii) elastic anisotropy. A discussion concerning microcracks and fluid content (saturation) issues follows. Experiments were performed on a Callovo-Oxfordian shale, Jurassic in age, recovered from a depth of $613 \mathrm{~m}$ in the eastern part of Paris basin in France.

\section{INTRODUCTION}

\subsection{Callovo-Oxfordian Shale}

Clayrocks, and shales in particular, represent approximately two-third of all sedimentary rocks. In oil and gas drilling operations, shales constitute $80 \%$ of all the drilled sections, mainly because they overlie most hydrocarbon bearing reservoirs. Furthermore, several countries are considering clayrocks as possible host lithologies for radioactive waste confinement, and therefore carrying out research programs to estimate feasibility of such solution. In this trend, the french agency for radioactive waste management, ANDRA, is evaluating the reliability of the CallovoOxfordian layer, Jurassic in age, located in the eastern part of France (Bure), at a depth ranging from $400 \mathrm{~m}$ to $700 \mathrm{~m}$. The static mechanical properties of this clayrock have been largely studied for the past few years (complete bibliography in Escoffier (2001)). However, very few velocity measurements, all at atmospheric pressure, were performed, and no velocity data at all under mechanical loading are available in the literature for this rock. The main outcome of interest here from these research programs is the transversely isotropic nature of Bure clayrock demonstrated experimentally in several labs for several locations and depths in the layer (David 2004). The main plane of symmetry is quasi-horizontal and corresponds to a very fine layering (bedding). This clayrock is then called a shale in the following.

\subsection{Shale Lithologies}

In general, the monitoring of elastic velocities in shales under mechanical loading are relatively uncommon in view of the abundance of this lithology in the shallow earth crustal rocks (Stanley and Christensen 2001). This relative rareness is partly due to: (i) the inherent difficulty of transmitting transducer signals through the waterproof pressure chamber in a loading apparatus ; and, on the other hand, (ii) the specificities in preparing and handling shale samples for mechanical testing (chemical sensitivity to water, extremely low permeability...). Most of the dynamic experimental studies reported in the literature on shale samples were performed under hydrostatic loading conditions (Johnston and Toksöz 1980; Jones and Wang 1981; Lo et al. 1986; Johnston and Christensen 1995; Hornby 1998; Stanley and Christensen 2001). Only Yin (1992) carried out triaxial tests.

\subsection{Main Goals}

The specific experimental setup available in our lab allows for the simultaneous measurement of five different velocities and two directions of strains on the same sample, under triaxial and pore pressurecontrolled conditions of loading. This procedure reduces the number of experiments on differently oriented samples usually needed to identify the dynamic properties of a rock. It also minimizes the errors due to the particular difference between two samples of 
the supposedly same lithology or in the same physical state (stress and saturation history since recovery from $613 \mathrm{~m}$ deep).

The main outcomes of this experiment are : (i) identification of the apparent dynamic stiffness tensor of the Callovo-Oxfordian shale from elastic velocity measurements ; (ii) assessment of velocity anisotropy, and its evolution under triaxial loading. This last step allows for the quantification of the intrinsic and stressinduced anisotropies, leading eventually to an estimation of the microcracks density and distribution evolutions in the shale sample under loading (Vernik 1994).

In the specific experiment described here, there are no strain data available during the hydrostatic loading because of experimental difficulties in strain gages gluing. However, an external LVDT allows for the estimation of the sample global axial strain during the deviatoric loading.

\section{EXPERIMENTAL PROCEDURE}

\subsection{Experimental Setup}

The rock physics group in the geology laboratory of the Ecole Normale Supérieure recently acquired a triaxial cell designed for the exploration of the hydromechanical behavior of shallow earth crustal rocks. This apparatus allows for pore pressure, hydrostatic and deviatoric stress to be applied independently on a cylindrical porous rock sample (up to $\varnothing 40 \mathrm{~mm} \times$ $L 80 \mathrm{~mm}$ ). The pore, confining and axial pressures may reach 100, 300 and $800 \mathrm{MPa}$ (on the largest samples), respectively. The originality of this loading cell is to allow for a maximum of 32 waterproof signal wires through the wall of the pressure chamber. The control and data acquisition are performed by means of a dedicated software designed in Labview ${ }^{\text {TM }}$ environment. The lab is temperature-controlled with an accuracy of $\pm 0.5^{\circ} \mathrm{C}$.

\subsection{Sample Description and Preparation}

The tested shale was provided by the french agency for radioactive waste management, ANDRA. The received large cores $(\varnothing 90 \mathrm{~mm} \times L 280 \mathrm{~mm})$ were recovered in 1995 from a depth of $613 \mathrm{~m}$ in the eastern part of Paris basin, near Bure, in France. These cores were since preserved in a so-called T1 cell, isolated from gas exchange with the storing environment, and maintained under small confining stress ( $\lesssim 1 \mathrm{MPa}$ ). The samples are dry-cored perpendicularly to the horizontal bedding, ground for parallel end faces, and preserved in waterproof membranes. In this specific experiment, the sample is $60 \mathrm{~mm}$ long with a $30 \mathrm{~mm}$ diameter. Few days before testing, during the phase of transducers gluing and curing on the sample, this one is maintained in a 100\% relative humidity (RH) environment in order to avoid drying. Usually, after putting it in $100 \% \mathrm{RH}$ atmosphere, the mass of the

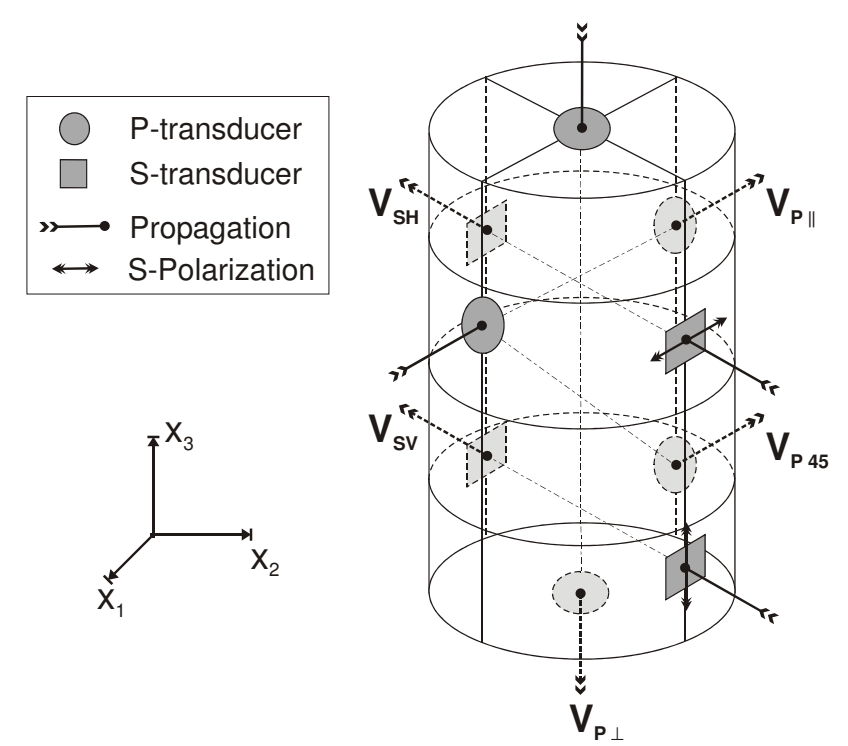

Figure 1: Piezoelectric transducers arrangement and velocity measurements on the shale sample.

sample increases then stabilizes. However, it is assumed that the sample may still be superficially unsaturated. It is tested under macroscopically undrained conditions within the loading cell.

\subsection{Transducers Arrangement}

For the experiment reported here, 22 signal wires were used. Both axial and circumferential strain gages and nine piezoelectric transducers were glued on the cylindrical sample. However, the strain gages signals are rapidly lost at the beginning of the test. The specific arrangement of piezoelectric transducers allows for the measurement of five different elastic velocities as shown in figure 1 . Indeed, let us define the reference frame $\left(x_{1}, x_{2}, x_{3}\right)$, with $\left(x_{1}, x_{2}\right)$ being the horizontal bedding plane of symmetry of the transversely isotropic cylindrical shale sample. Then, measurements of three compressional velocities and two shear velocities, in three different directions are provided. These velocities are referenced with respect to the bedding plane, i.e., $V_{P}\left(0^{\circ}\right)$ for the bedding-parallel compressional velocity, $V_{P}\left(45^{\circ}\right)$ for the $45^{\circ}$-to-bedding compressional velocity, $V_{P}\left(90^{\circ}\right)$ for the bedding-perpendicular compressional velocity, $V_{S H}\left(0^{\circ}\right)$ for the horizontally polarized, beddingparallel shear velocity, and $V_{S V}\left(0^{\circ}\right)$ for the vertically polarized, bedding-parallel shear velocity. In the case of transverse anisotropy, this latter is equal to the shear wave velocity $V_{S}\left(90^{\circ}\right)$ perpendicular to the bedding. Note that the specifically designed compressional piezoelectric transducers are virtually load insensitive. On the other hand, the shear piezoceramics are simply glued to a fitting metal piece, itself glued on the sample, and are therefore load sensitive. 


\subsection{Measurement Technique}

For velocity measurement, the classical Ultrasonic Pulse Transmission technique is used (Birch 1960; Tosaya 1982; Yin 1992). This method consists in measuring the travel time of a solitary elastic pulse through the rock sample of known travelling wave path length. The frequency of the solitary pulse in this experiment is $1 \mathrm{MHz}$.

\subsection{Test Conditions \& Loading Path}

The chemical sensitivity of this shale to external water and its very low permeability precluded any pore pressure control of the test. Therefore, the tests are performed in macroscopically undrained conditions within the loading cell. The in-situ stress is estimated from depth and rock density. Indeed, we assume hydrostatic state of stress in this large sedimentary basin, neglecting any deviatoric stress possibly due to tectonic stress build-up. This in-situ stress is estimated around $15 \mathrm{MPa}$.

The loading starts with confining pressure cycle between 0 and $55 \mathrm{MPa}$, i.e., (i) loading from 0 to $20 \mathrm{MPa}$; (ii) unloading from 20 to $3 \mathrm{MPa}$; (iii) reloading from 3 to $55 \mathrm{MPa}$; then (iv) unloading from 55 to $15 \mathrm{MPa}$. The loading rate is $0.013 \mathrm{MPa} / \mathrm{s}$ during the pressure-controlled hydrostatic loading. After the fourth stage, a deviatoric stress is applied and increased until sample rupture. The deviatoric loading is displacement-controlled in order to avoid sudden sample failure. The velocity measurements are made after stabilization of all sample parameters at a given loading stage.

\section{EXPERIMENTAL RESULTS}

\subsection{Error Analysis}

For this particular experiment, corrections due to the rock deformation in the estimation of the velocity change during the hydrostatic loading are not feasible with accuracy because of a lack of strain measurement. However, a previous experiment on a similar sample, under the same conditions, allowed for strain data acquisition. Therefore, in the hydrostatic part of the present experiment, these strain data are used to correct velocity data. Both samples where cored exactly at the same depth, one centimeter far from each other, and no apparent heterogeneity was noted.

Furthermore, a complete error analysis of the Ultrasonic Pulse Transmission technique has been carried out Yin (1992) and Hornby (1998). This analysis includes the errors in the travel time picks for the measured and reference travel times, and the error in the sample size measurements. In our experimental case, using Hornby (1998) equation (6), the relative error reaches a maximum of $1.1 \%$ in the velocity measurements, depending on the wave type and propagation direction.

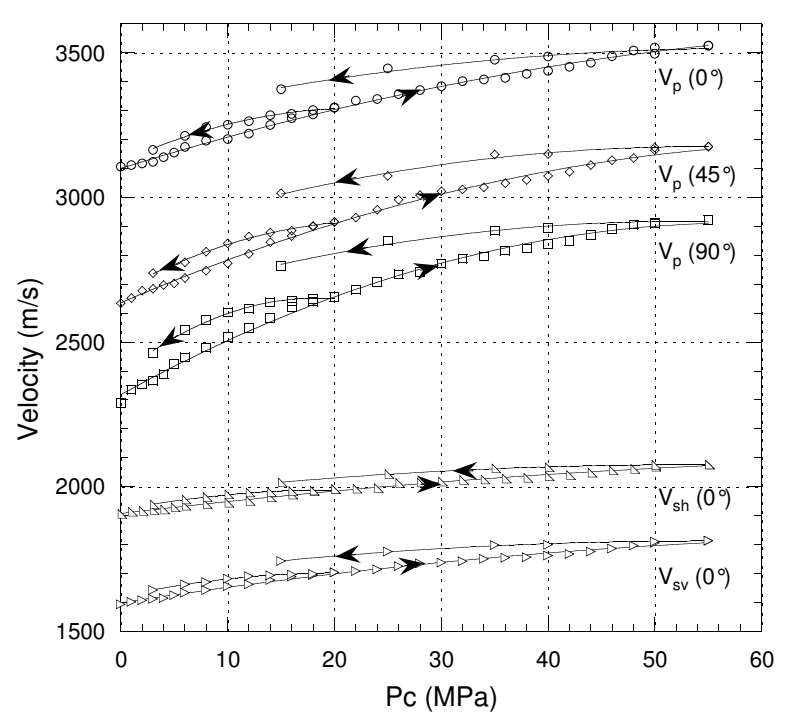

Figure 2: Elastic wave velocities evolution under hydrostatic loading.

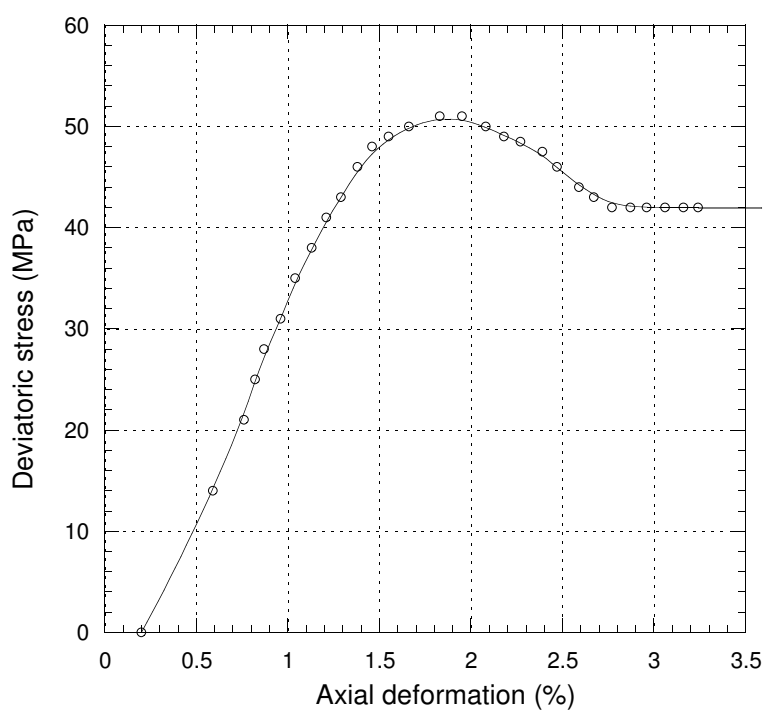

Figure 3: Axial stress - axial strain curve.

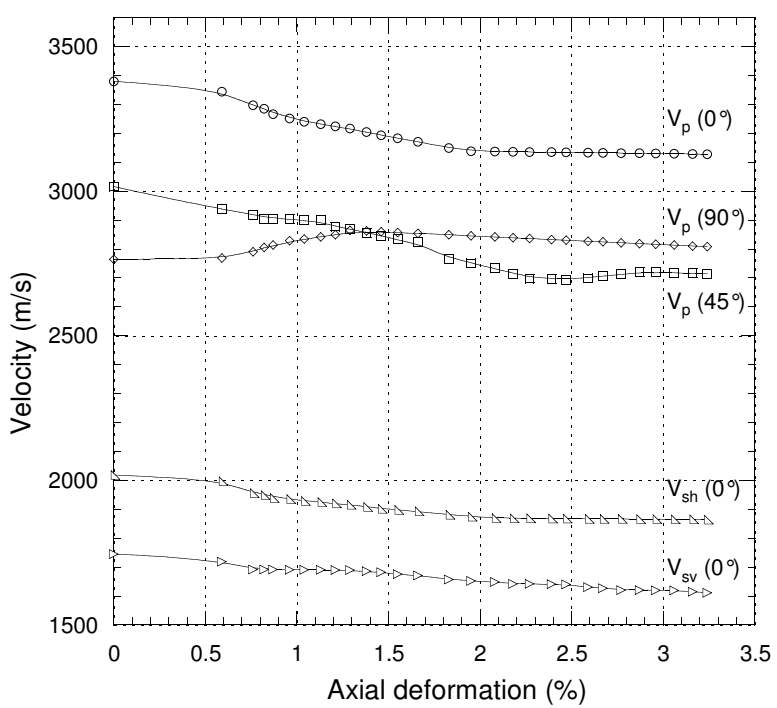

Figure 4: Elastic wave velocities evolution under deviatoric loading, at $15 \mathrm{MPa}$ confining pressure. 


\subsection{Experimental Data}

Figure 2 shows the evolution of the five measured velocities $V_{P}\left(0^{\circ}\right), V_{P}\left(45^{\circ}\right), V_{P}\left(90^{\circ}\right), V_{S H}\left(0^{\circ}\right)$ and $V_{S V}\left(0^{\circ}\right)$, under confining pressure varying between 0 and $55 \mathrm{MPa}$. All velocities increase with increasing pressure, and a hysteresis effect exists upon pressure release at 20 and $55 \mathrm{MPa}$. Note that in the $0-$ $55 \mathrm{MPa}$ pressure range, $\Delta_{p} V_{P}\left(0^{\circ}\right)<\Delta_{p} V_{P}\left(45^{\circ}\right)<$ $\Delta_{p} V_{P}\left(90^{\circ}\right)$. Even the amplitudes of the hysteresis velocity lags are ordered in the same way, i.e., $\Delta_{h} V_{P}\left(0^{\circ}\right)<\Delta_{h} V_{P}\left(45^{\circ}\right)<\Delta_{h} V_{P}\left(90^{\circ}\right)$.

Figure 3 shows the axial stress-axial strain curve during the deviatoric part of the loading path. The sample failure happened probably around the peak axial stress (51 MPa), and for a $1.9 \%$ axial strain.

Figure 4 shows the evolution of the five measured velocities $V_{P}\left(0^{\circ}\right), V_{P}\left(45^{\circ}\right), V_{P}\left(90^{\circ}\right), V_{S H}\left(0^{\circ}\right)$ and $V_{S V}\left(0^{\circ}\right)$, under this deviatoric loading. All velocities decrease with increasing axial stress except $V_{P}\left(0^{\circ}\right)$ which first increases until $1.3 \%$ axial strain, then reduces slightly.

\section{THEORETICAL CONSIDERATIONS}

\subsection{Dynamic Stiffness Matrix}

\subsubsection{Definition}

Transversely isotropic materials (hexagonal symmetry) may be adequately described in the elastic regime by five independent stiffness constants relating stress to strain components. They form a fourth order tensor which may be written in the Voigt notation as a twelve components matrix in the reference frame $\left(x_{1}, x_{2}, x_{3}\right)$, i.e.,

$$
\left[\begin{array}{cccccc}
C_{11} & C_{12} & C_{13} & 0 & 0 & 0 \\
C_{12} & C_{11} & C_{13} & 0 & 0 & 0 \\
C_{13} & C_{13} & C_{33} & 0 & 0 & 0 \\
0 & 0 & 0 & C_{44} & 0 & 0 \\
0 & 0 & 0 & 0 & C_{44} & 0 \\
0 & 0 & 0 & 0 & 0 & C_{66}
\end{array}\right]
$$

where $C_{12}=C_{11}-2 C_{66}$.

When evaluated from elastic wave velocity measurements, this tensor characterizes the dynamic apparent behavior of the material. These five independent $C_{i j}$ constants may be related uniquely to the five classical Young's, Poisson's and shear dynamic moduli, i.e., $E_{1}^{d}, E_{3}^{d}, \nu_{12}^{d}, \nu_{13}^{d}$ and $\mu_{13}^{d}$ for instance.

These five independent $C_{i j}$ constants are related to the five elastic wave velocities measured experimentally as follows :

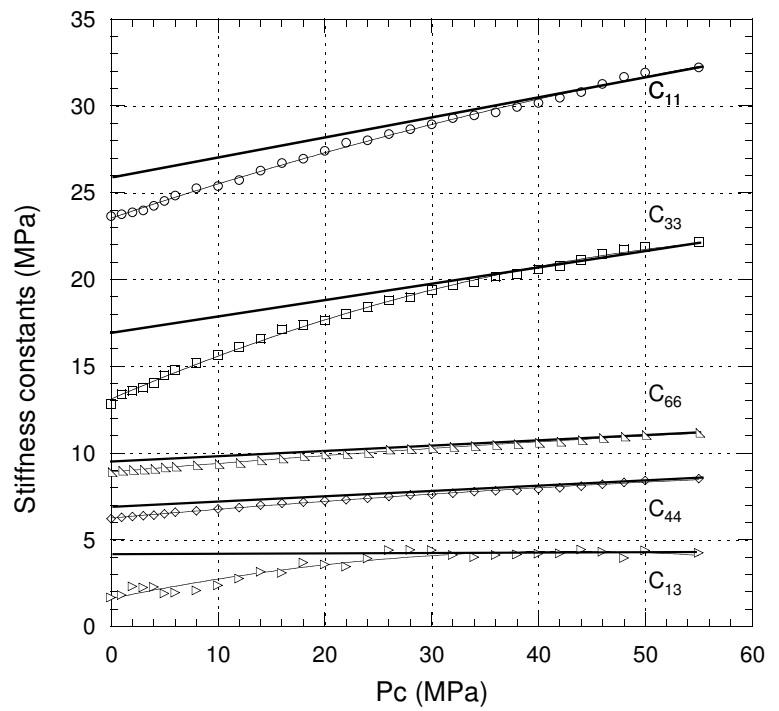

Figure 5: Dynamic stiffness parameters evolution under hydrostatic loading.

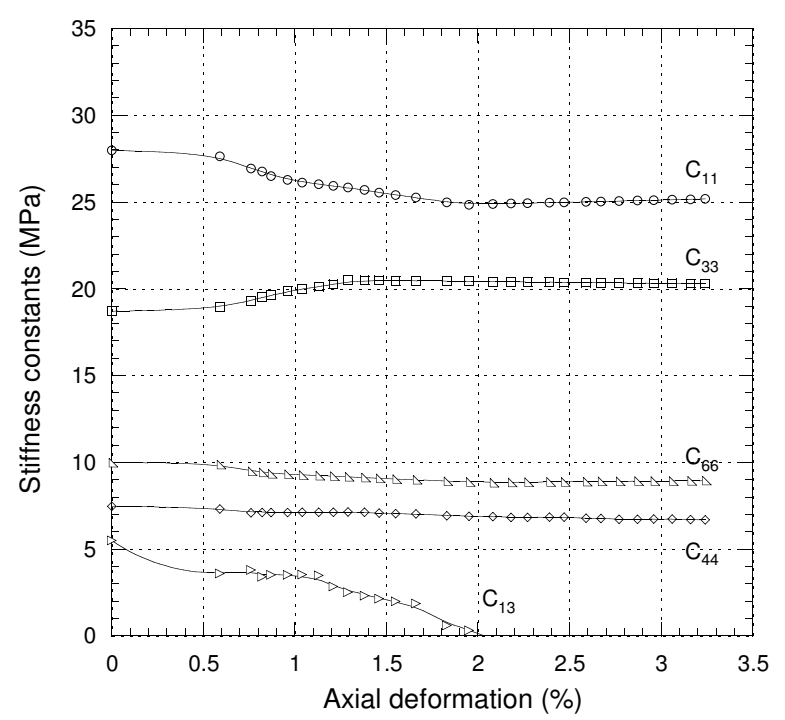

Figure 6: Dynamic stiffness parameters evolution under deviatoric loading, at $15 \mathrm{MPa}$ confining pressure.

$$
\begin{aligned}
C_{11}= & \rho V_{P}^{2}\left(0^{\circ}\right), \quad C_{33}=\rho V_{P}^{2}\left(90^{\circ}\right), \\
C_{44}= & \rho V_{S V}^{2}\left(0^{\circ}\right), \quad C_{66}=\rho V_{S H}^{2}\left(0^{\circ}\right), \\
C_{13}= & -C_{44}+\left[\left(C_{11}+C_{44}-2 \rho V_{P}^{2}\left(45^{\circ}\right)\right)\right. \\
& \left.\left(C_{33}+C_{44}-2 \rho V_{P}^{2}\left(45^{\circ}\right)\right)\right]^{1 / 2} .
\end{aligned}
$$

\subsubsection{Evolution under Loading}

Figure 5 and 6 illustrate the evolution of the dynamic stiffness parameters $C_{i j}$ with increasing confining pressure and axial stress, respectively. Note that figure 5 allows for the estimation of the crack-free dynamic moduli at low pressure. 


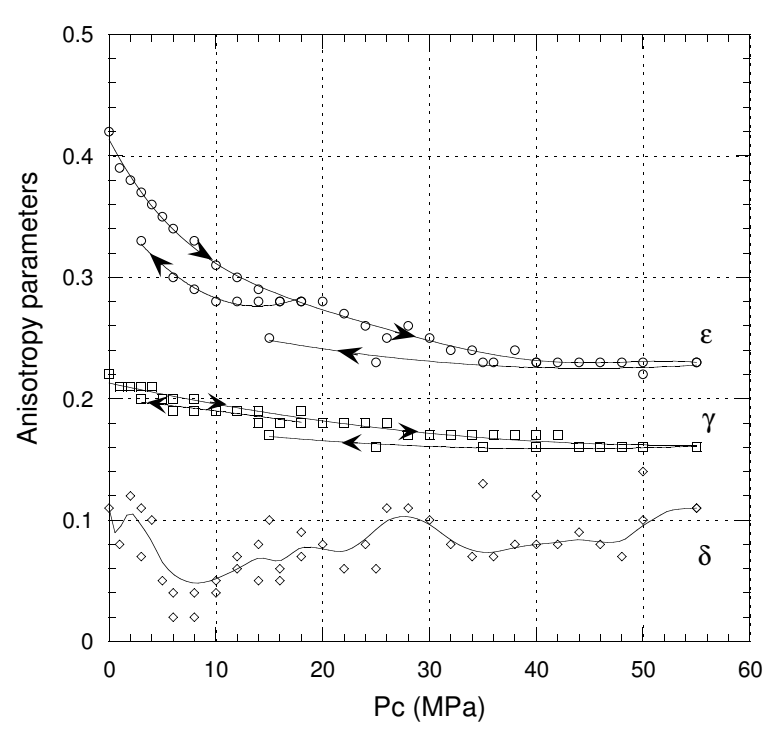

Figure 7: Anisotropy parameters evolution under hydrostatic loading.

\subsection{Anisotropy Parameters}

\subsubsection{Definition}

In the specific context of transverse isotropy analysis, it is convenient to express three of the five dynamic elastic constants in (1) in terms of three anisotropy parameters, initially introduced by Thomsen (1986), i.e.,

$$
\begin{aligned}
& \varepsilon=\frac{C_{11}-C_{33}}{2 C_{33}}, \quad \gamma=\frac{C_{66}-C_{44}}{2 C_{44}}, \\
& \delta=\frac{\left(C_{13}+C_{44}\right)^{2}-\left(C_{33}-C_{44}\right)^{2}}{2 C_{33}\left(C_{33}-C_{44}\right)} .
\end{aligned}
$$

where $\varepsilon$ measures the P-wave anisotropy, $\gamma$ measures the $\mathrm{S}$-wave anisotropy and $\delta$ is totally independent of horizontal velocity and may be either positive or negative for geomaterials (Thomsen 1986; Hornby 1998). The main advantage of these parameters is their dimensionless nature, therefore facilitating qualitative insight into the material behavior.

\subsubsection{Evolution under Loading}

Figure 7 and 8 show the evolution of velocity anisotropy parameters $\varepsilon, \gamma$ and $\delta$ under hydrostatic and deviatoric loadings, respectively. Note the constant value of $\delta$ under hydrostatic loading and its drastic decrease until $2.25 \%$ axial strain and then stabilization at a negative value under deviatoric load.

\section{PHYSICAL INTERPRETATION: DISCUSSION}

\subsection{Anisotropy Issue}

The main factors contributing to velocity anisotropy of rocks are: (i) preferred orientation of void space (pores and cracks) ; (ii) textural-structural features (bedding or foliation) ; and (iii) anisotropic constituent minerals. Crack-induced anisotropy reduces

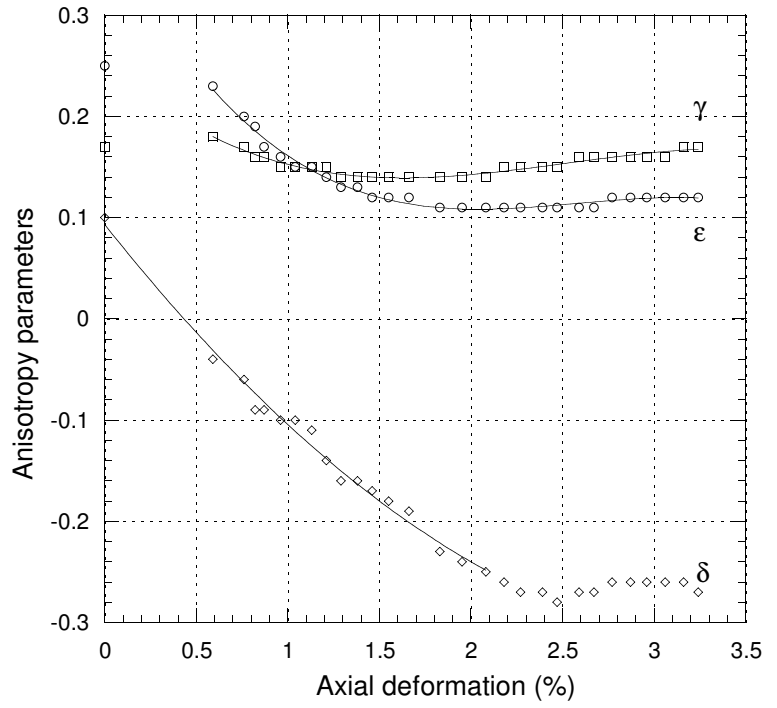

Figure 8: Anisotropy parameters evolution under deviatoric loading, at $15 \mathrm{MPa}$ confining pressure.

with increasing hydrostatic load, leading eventually to the estimation of the intrinsic anisotropy at high confining pressure, due to factors (ii) and (iii) only. If we assume that crack-induced (subscript $c$ ) and intrinsic (subscript $i$ ) anisotropies are additive (Vernik and Liu 1997), we may extract the intrinsic anisotropies $\varepsilon_{i}$ and $\gamma_{i}$ from Fig. 7 at $55 \mathrm{MPa}$, i.e.,

$$
\begin{aligned}
\varepsilon_{i} & =0.23, \\
\gamma_{i} & =0.16 .
\end{aligned}
$$

This allows for the estimation of the crack-induced anisotropy variation under hydrostatic loading, i.e., $\Delta_{p} \varepsilon_{c}=0.19$ and $\Delta_{p} \gamma_{c}=0.05$. The $\delta$ parameter seems constant under hydrostatic loading, i.e., $\delta \simeq 0.08$. However, it is very sensitive to the deviatoric load, and decreases drastically with the increase of this latter to a value of $\delta_{c}=-0.28$ (Fig.8). Furthermore, under deviatoric loading, both $\varepsilon$ and $\gamma$ anisotropy parameters decrease until $1.3 \%$ axial strain, then stabilize at $\varepsilon_{d}=0.11$ and $\gamma_{d}=0.14$. After, $1.9 \%$ axial strain, $\gamma$ increases back. We therefore deduce that after closing the bedding-parallel microcracks under confining pressure, the deviatoric loading induces a damage to which the horizontal S-velocities are sensitive through the $\gamma$ parameter. This damage may further be interpreted in terms of bedding-perpendicular microcracks. Note that for $\varepsilon$ parameter, the crackinduced and intrinsic anisotropies are of the same order of magnitude, which confirms the importance of charaterizing the microcracked state of shales through anisotropy analysis.

The global analysis of the anisotropy data leads to a conceptual modeling of the microcracking history of a shale after coring and recovery. In general, (i) stress relief, (ii) microhydraulic fracturing, (iii) bottomhole stressing, (iv) dessication, and (v) in-situ mi- 
crocracks, contribute to the final state of microcracking in a rock sample in the lab. For this shale, microcracks close under confining pressure, preferentially in the direction perpendicular to the bedding (beddingparallel microcracks). The deviatoric loading induces damage (bedding-perpendicular microcracks).

\subsection{Fluid Content and Saturation Issue}

As pointed out by Jones and Wang (1981), the partial saturation of the sample manifests itself by the velocity hysteresis under cyclic confining pressure. They noticed that this hysteresis is drastically reduced when the shale sample is rehumidified, and even cancels at very high confining pressure (400 MPa). Indeed, at ambient pressure, the void space is partially gas-saturated, in particular at the boundaries of the sample where gas exchange with atmosphere may occur (surface drying). When submitted to a hydrostatic load, the microcracks closure reduces the void space, which is eventually totally occupied by the pore water at very high confining pressure. At this stage, hysteresis due to partial fluid saturation is observable. In our experiment, it seems that even at $55 \mathrm{MPa}$ confining pressure, hysteresis still exists, which allows us to assume that even at this pressure value, the pore space is still not fully saturated by water.

This brings out the question of effective pressure in the sample under loading. We will assume that during the hydrostatic loading, the confining pressure is equal to the effective pressure, since the pore space is not water-saturated and the incompressibility of gas is negligible compared to that of liquid. The sample acts as under drained conditions.

Two other phenomena related to solid/fluid interactions are likely to occur in shales: (i) chemical softening as a result of fluid-clay interaction and swelling (smectites in particular) ; and (ii) physicochemical softening due to lubrication of grain interfaces. These issues are responsible for the inherent difficulty of shale experimental testing.

\section{CONCLUDING REMARKS}

Anisotropic elastic properties of a quasi-saturated Jurassic shale under macroscopically undrained conditions were determined by means of ultrasonic velocity measurements. These five velocity measurements were performed on the sample while under hydrostatic and deviatoric loadings in a triaxial cell. The experimental method is based on a single core test, minimizing the errors due to the particular difference between two samples of the supposedly same lithology or in the same physical state (stress and saturation history since recovery).

Analysis and quantification of anisotropy may lead to the estimation of critical parameters such as microcracks density and orientation, as well as crack aspect ratio, using Hudson's or Kachanov's models. This type of experiment will provide with input parameters for modeling wave propagation in porous, fluidinfiltrated, very low permeability shales. A natural way to model such medium is to use Biot's dynamic theory of poroelasticity, possibly extended to account for squirt-flow phenomenon due to the existence of an equant porosity connected to an anisotropic one (crack-shape).

\section{ACKNOWLEDGEMENTS}

Support and shale samples for this research program have been provided by the french agency for radioactive waste management, ANDRA. This support is gratefully acknowledged. I would also like to thank Guy Marolleau for his crucial help in the setting of the experiment in the triaxial cell.

\section{REFERENCES}

Birch, F. (1960). The velocity of compressional waves in rocks to 10 kilobars. J. Geophys. Res. 65, 1083-1102.

David, C. (2004). Personal Communication.

Escoffier, S. (2001). Caractérisation Expérimentale du Comportement Hydromécanique des Argilites de Meuse/Haute-Marne. Ph. D. thesis, Institut National Polytechnique de Lorraine, Nancy, France.

Hornby, B. E. (1998). Experimental laboratory determination of the dynamic elastic properties of wet, drained shales. J. Geophys. Res. 103(B12), 29945-29964.

Johnston, D. H. and M. N. Toksöz (1980). Ultrasonic P and S wave attenuation in dry and saturated rocks under pressure. J. Geophys. Res. 85(B2), 925-936.

Johnston, J. E. and N. I. Christensen (1995). Seismic anisotropy of shales. J. Geophys. Res. 100(B4), 59916003.

Jones, L. E. A. and H. F. Wang (1981). Ultrasonic velocities in cretaceous shales from the williston basin. Geophysics 46(3), 288-297.

Lo, T.-W., K. B. Coyner, and M. N. Toksöz (1986). Experimental determination of elastic anisotropy of berea sandstone, chicopee shale, and chelmsford granite. Geophysics 51(1), 164-171.

Stanley, D. and N. I. Christensen (2001). Attenuation anisotropy in shale at elevated confining pressures. Int. J. Rock Mech. Min. Sci. 38, 1047-1056.

Thomsen, L. (1986). Weak elastic anisotropy. Geophysics 51(10), 1954-1966.

Tosaya, C. A. (1982). Acoustical Properties of Clay-Bearing Rocks. Ph. D. thesis, Stanford University, Stanford, USA.

Vernik, L. (1994). Hydrocarbon-generaion-induced microcracking of source rocks. Geophysics 59(4), 555-563.

Vernik, L. and X. Liu (1997). Velocity anisotropy in shales: A petrophysical study. Geophysics 62(2), 521-532.

Yin, H. (1992). Acoustic Velocity and Attenuation of Rocks: Isotropy, Intrinsic Anisotropy, and Stress Induced Anisotropy. Ph. D. thesis, Stanford University, Stanford, USA. 